\title{
Status and First Results of the Acoustic Detection Test System AMADEUS
}

\author{
Robert Lahmann on behalf of the ANTARES Collaboration* \\ Erlangen Centre for Astroparticle Physics (ECAP), Erwin-Rommel-Str. 1, 91058 Erlangen, GERMANY
}

\begin{abstract}
The AMADEUS system is integrated in the ANTARES neutrino telescope in the Mediterranean Sea and aims for the investigation of acoustic particle detection techniques in the deep sea. Installed at a depth of more than $2000 \mathrm{~m}$, the acoustic sensors of AMADEUS are using piezo-ceramic elements for the broad-band recording of acoustic signals with frequencies ranging up to $125 \mathrm{kHz}$. AMADEUS consists of six clusters, each one comprising six acoustic sensors that are arranged at distances of roughly $1 \mathrm{~m}$ from each other. Three acoustic clusters are installed along a vertical mechanical structure (a so-called Line) of ANTARES with spacings of about $15 \mathrm{~m}$ and $110 \mathrm{~m}$, respectively. The remaining 3 clusters are installed with vertical spacings of $15 \mathrm{~m}$ on a further Line of the ANTARES detector. The horizontal distance between the two lines is $240 \mathrm{~m}$. Each acoustic cluster allows for the suppression of random noise by requiring local coincidences and the reconstruction of the arrival direction of acoustic waves. Source positions can then be reconstructed using the precise time correlations between the clusters provided by the ANTARES clock system. AMADEUS thus allows for extensive acoustic background studies including signal correlations on several length scales as well as source localisation. The system is therefore excellently suited for feasibility studies for a potential future large scale acoustic neutrino telescope in sea water. Since the start of data taking on December 5th, 2007 a wealth of data has been recorded. The AMADEUS system will be described and some first results will be presented.
\end{abstract}

Key words: AMADEUS, ANTARES, neutrino telescope, acoustic neutrino detection, thermo-acoustic model PACS: 95.55.Vj, 95.85.Ry, 13.15.+g, 43.30.+m

\section{Introduction}

The production of pressure waves by cascades of fast particles passing through liquids was predicted as early as 1957 [1], leading to the development of the so-called thermo-acoustic model in the 1970s $[2,3]$. According to the model, the energy deposition of charged particles in liquids leads to a local heating of the medium which can be regarded as instantaneous with respect to the typical time scale of the acoustic signals. Because of the temperature change the medium expands or contracts according to its volume expansion coefficient $\alpha$. The accelerated mo-

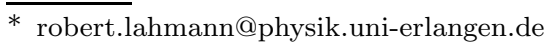

tion of the heated medium produces a pressure pulse which propagates through the medium.

Fig. 1 shows a bipolar pressure pulse from a recent Monte Carlo simulation of neutrino interactions in water for a hadronic cascade with an energy of $1 \mathrm{EeV}$ [4]. The amplitude was calculated at a distance of $1 \mathrm{~km}$ from the cascade in a plane perpendicular to the shower axis at the shower maximum.

The cascades extend over a length of a few metres with a radius of only a few $\mathrm{cm}$. In radial direction, i.e. perpendicular to the shower axis, the coherent superposition of the emitted sound waves leads to the propagation of the sound within a flat disk (often referred to as pancake) with a small opening angle. With a linear energy dependence, the pressure pulse 


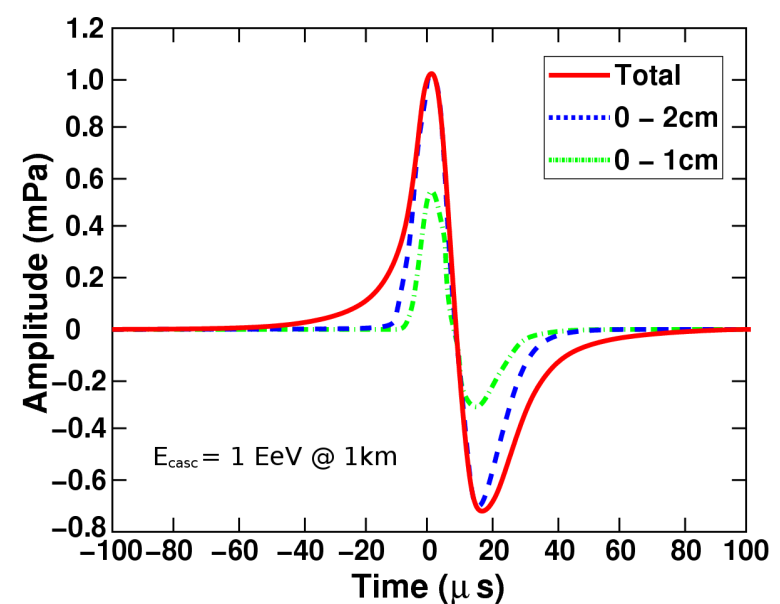

Fig. 1. Simulated bipolar pressure pulse from a $10^{18} \mathrm{eV}$ hadronic cascade at a radial distance of $1 \mathrm{~km}$. Roughly half of the pressure pulse is produced within a radius of $1 \mathrm{~cm}$ (dash-pointed line) of the cascade, whereas the energy distribution within a radius of $2 \mathrm{~cm}$ (dashed line) is nearly completely responsible for the final signal shape (solid line). Figure adapted from [4].

amplitude scales as

$p(1 \mathrm{~km}) \approx 1 \mathrm{mPa} \cdot \frac{E_{\text {casc }}}{1 \mathrm{EeV}}$

with the spectral energy density peaking around $10 \mathrm{kHz}$.

Two major advantages over an optical neutrino telescope make acoustic detection worth studying. First, the attenuation length is on the order of $5 \mathrm{~km}$ $(1 \mathrm{~km})$ for $10 \mathrm{kHz}(20 \mathrm{kHz})$ signals. This is approximately one order of magnitude higher than for Cherenkov light (attenuation length $60 \mathrm{~m}$ or below) in the relevant frequency band.

The second advantage is the much simpler sensor design and readout electronics required for acoustic measurements: No high voltage is required in the case of acoustic measurements and time scales are in the $\mu$ s range for acoustics as compared to the ns range for optics. This allows for online implementation of advanced signal processing techniques. Efficient data filters are essential, as the signal amplitude is relatively small compared to the acoustic background in the sea, which complicates the unambiguous determination of the signal.

Understanding the background conditions at the site of a potential large-scale acoustic neutrino detector is therefore crucial in order to assess the feasibility of such a device. It is important to realize that there are two kinds of background which need to be understood: First, there is ambient noise which can be described by its characteristic power spectral density. This noise is determined by environmental processes and will be discussed in Sec. 5. This background defines the minimum pulse heights that can be measured if a given signal-to-noise ratio can be achieved with a search algorithm. To measure this background, in principle one hydrophone is sufficient and the synchronisation among multiple hydrophones is not crucial.

Second, there are neutrino-like events, i.e. signals which have the characteristic bipolar pulse shape shown in Fig. 1, but have a different origin. It is important to measure their spatial, temporal and pulse-height distribution in order to assess the probability for random coincidences that mimic the characteristic pancake structure of a neutrino sound wave. For this kind of measurement, a hydrophone array is required and the synchronisation among the hydrophones is crucial.

A huge number of signals recorded by AMADEUS are emitted by marine mammal, mainly dolphins, which is of great interest to marine biologists. The prospective research activities in this area will however not be addressed in this paper.

In the following section, an overview of the AMADEUS System will be given (Sec. 2), which will be followed by a description of the components of the system in Sec. 3. The system response as measured in the laboratory prior to the deployment will be discussed in Sec. 4 and some first results will be discussed in Sec. 5 .

\section{Overview of the AMADEUS System}

\subsection{Goals}

It is the declared main goal of the AMADEUS project to perform a feasibility study for a potential future large-scale acoustic neutrino detector. To this end, the following aims are pursued:

- Long term background investigations (rate of neutrino-like signals, localisation of sources, levels of ambient noise);

- Investigation of signal and background correlations on different length scales;

- Development and tests of filter and reconstruction algorithms;

- Tests of different sensors and sensing methods;

- Studies of hybrid detection methods.

These goals were driving the design of the AMADEUS system, which will now be discussed. 


\subsection{AMADEUS as Part of ANTARES}

AMADEUS is a part of the ANTARES neutrino telescope [5] in the Mediterranean Sea, which was completed on May 30th, 2008. A sketch of the detector is shown in Fig. 2. ANTARES is located in the deep sea, about $40 \mathrm{~km}$ south of Toulon, at a water depth of about $2500 \mathrm{~m}$. It comprises 12 vertical structures, the detection lines (or lines for short) plus a 13th line, called Instrumentation Line or $I L$, which is equipped with instruments for monitoring the environment of the detector. Each detection line holds 25 storeys that are arranged at equal distances of $14.5 \mathrm{~m}$ along the line, interlinked by electro-mechanical-optical cables. A standard storey consists of a titanium support structure, holding three Optical Modules (OMs), i.e. photomultiplier tubes (PMTs) inside glass spheres with a nominal diameter of $432 \mathrm{~mm}$, and one Local Control Module ( $L C M)$. The LCM holds the offshore electronics and the power supply within a cylindrical titanium container (cf. Sec. 3.2). Each line is fixed on the sea floor by an anchor (Bottom String Socket, BSS) and held vertically by a buoy.

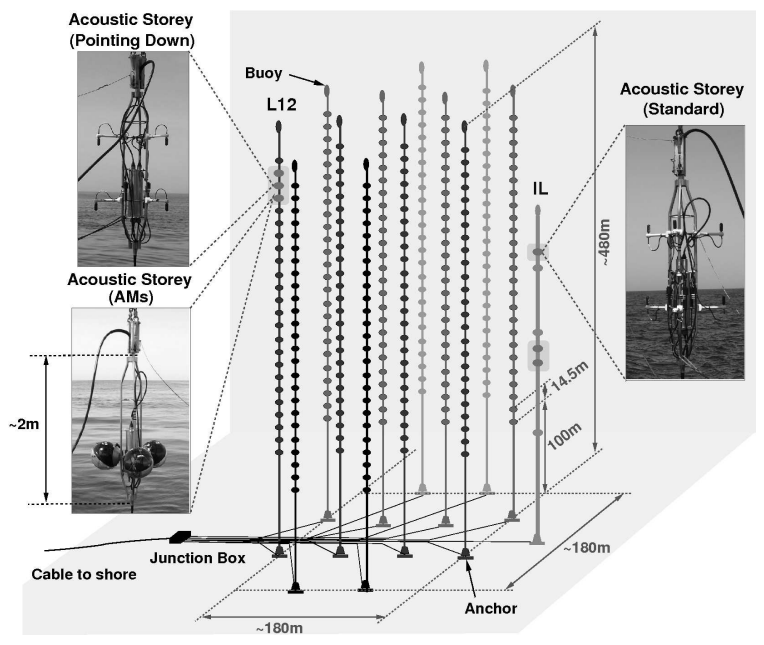

Fig. 2. A sketch of the ANTARES detector. The six Acoustic Storeys are highlighted and their three different setups are shown.

Acoustic sensing was integrated in form of Acoustic Storeys which are modified versions of standard ANTARES storeys: Acoustic sensors replace the OMs and custom-designed electronics is used to digitise and preprocess the analogue signals. Details will be discussed below.

The three Acoustic Storeys on the IL started data taking when the connection to shore was established on Dec. 5th, 2007. The Acoustic Storeys on Line 12 (L12) were connected to shore during the completion of ANTARES in May 2008. AMADEUS is now fully functional with 34 of its 36 sensors working.

\subsection{Description of the System}

It has been a fundamental design principle of the AMADEUS system to make use of standard ANTARES hard- and software as much as possible. The intention was to minimise the effort for design and engineering and to reduce the failure risks for both ANTARES and AMADEUS by reducing the need for additional quality assurance and control measures to a minimum. In order to integrate the system successfully into the ANTARES detector, design efforts in three basic areas were necessary: First, the development of acoustic sensors that replace the OMs of standard ANTARES storeys; second, the development of an offshore acoustic $\mathrm{ADC}$ and preprocessing electronics board; third, the development of on- and offline software. These subjects will be discussed in more detail in Sections $3.1,3.3$ and 3.4, respectively.

Each Acoustic Storey is equipped with six acoustic sensors with interspacings on the order of $1 \mathrm{~m}$. The Acoustic Storeys on the IL are located at $180 \mathrm{~m}$, $195 \mathrm{~m}$, and $305 \mathrm{~m}$ above the sea floor, respectively. Line 12 is anchored at a horizontal distance of about $240 \mathrm{~m}$ from the IL, with the Acoustic Storeys positioned at heights of $380 \mathrm{~m}, 395 \mathrm{~m}$, and $410 \mathrm{~m}$. With this setup, the maximum distance between two Acoustic Storeys is $340 \mathrm{~m}$. Two of the six Acoustic Storeys are shown in Fig. 3.

The final system has the full capabilities of a detector, such as time synchronisation and a continuously operating data acquisition, and is scalable to a larger number of Acoustic Storeys. It combines local clusters of acoustic sensors (the Acoustic Storeys) with large cluster spacings, allowing for fast direction reconstruction with individual storeys that then can be combined to reconstruct the position of a source.

\section{Components of the AMADEUS System}

\subsection{The Acoustic Sensors}

Two types of acoustic sensors are used in AMADEUS: hydrophones and so-called Acoustic Modules (AMs, cf. Fig. 3). In both cases, the sensors 

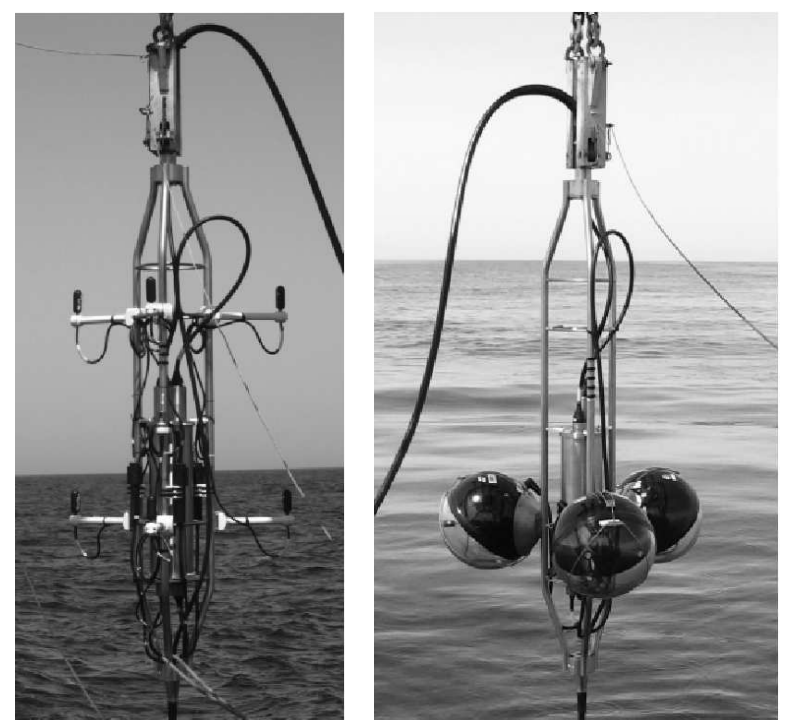

Fig. 3. An Acoustic Storey of the IL (left), equipped with hydrophones, and the lowermost Acoustic Storey of L12 (right) equipped with Acoustic Modules, shown during their deployment.

are based on piezo-electrical ceramic elements that convert pressure waves into voltage signals, which are then amplified for readout [6]. The ceramics and amplifiers are coated in polymer plastics in the case of the hydrophones. Those sensors have a diameter of about $4 \mathrm{~cm}$ and a length (from the cable junction to the opposite end) of about $10 \mathrm{~cm}$.

For the AMs, the ceramics are glued to the inside of the same spheres used for the Optical Modules of ANTARES. This design was inspired by the idea to investigate an option for acoustic sensing that can be combined with a PMT in the same housing.

In order to obtain a complete $2 \pi$-coverage of the azimuthal angle $\phi$, the 6 sensors are distributed over the $3 \mathrm{AMs}$ of the storey within a plane perpendicular to the longitudinal axis of a storey. The two piezo elements within one sphere are arranged at an opening angle of $60^{\circ} 1$

The three Acoustic Storeys on the IL house hydrophones only, whereas the lowermost storey of Line 12 holds AMs (cf. Fig. 2). The standard mounting of the hydrophones is with their cable junction pointing downwards. In one of the storeys on Line 12 , the hydrophones were mounted with the cable junction, where the sensitivity is largely reduced,

\footnotetext{
1 For reasons such as limited data rate and the pre-existing design of the ANTARES offshore electronics, implementing more than 2 sensors per AM would have increased the technical effort disproportionately.
}

pointing upwards. This allows for investigations of the directionality of background from ambient noise, which is expected to come mainly from the sea surface.

Three of the five storeys holding hydrophones are equipped with commercial hydrophone 2 and the other two with hydrophones developed and produced at the Erlangen Centre for Astroparticle Physics (ECAP).

All acoustic sensors are tuned to have a low noise level and to be sensitive over the whole frequency range of interest from 1 to $50 \mathrm{kHz}$. Their typical sensitivity is around $-145 \mathrm{~dB}$ re. $1 \mathrm{~V} / \mu \mathrm{Pa}$ (including preamplifier) [7].

\subsection{Offshore Electronics and Acoustic Data Acquisition}

In the ANTARES DAQ scheme [8], the digitisation of sensor signals is conducted within the offshore electronics container on each storey by several custom-designed electronics boards, which send all digitised data to shore. Here data reduction is performed and the data is searched for events of interest. With its capability of timing synchronisation on the nanosecond-scal 3 and transmission of several MByte per second and per storey, it is perfectly suited for the acquisition of acoustic data. In addition, in each LCM a Compass board measures the tilt and the orientation of the storey.

For the digitisation and preprocessing of the acoustic signals and for feeding them into the ANTARES data stream, the AcouADC board was designed. Each board processes the differential signals from two acoustic sensors, which results in a total of three such boards per storey.

Figure 4 shows the fully equipped LCM of an Acoustic Storey. All components except for the AcouADC boards are standard components that are present in every LCM crate of ANTARES.

\subsection{The AcouADC Board}

The AcouADC board is shown in Fig. 5. It consists of an analogue and a digital part. The analogue

\footnotetext{
2 Custom produced by High Tech Inc (HTI) in Gulfport, MS (USA).

3 The clock system is in fact capable of providing subnanosecond precision for the synchronisation of the optical data recorded by the PMTs. This precision, however, is not required for the acoustic data.
} 


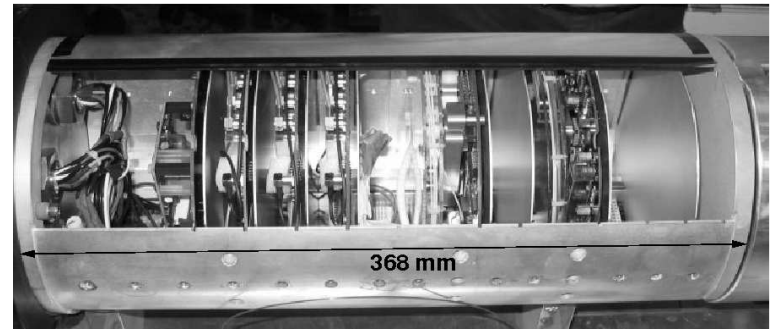

Fig. 4. An LCM crate equipped with AcouADC boards before insertion into its titanium housing. From the left to the right, the following boards are installed: The Compass board; 3 AcouADC boards; a Data Acquisition (DAQ) board that sends the data to shore; and a Clock board that provides the timing signals to correlate measurements performed in different storeys.

part amplifies the voltage signals coming from the acoustic sensors by one of 12 adjustable factors between 1 and 562 and applies a bandpass filter to the resulting signal. To protect the analogue part from potential electromagnetic interference, it is shielded by metal covers. The system has low noise and is designed to be - together with the sensors - sensitive to the acoustic background of the deep sea over a wide frequency-range. The dynamic range achieved for the standard gain factor of 10 is from about $5 \mathrm{mPa}$ to $5 \mathrm{~Pa}$ in peak-to-peak amplitude of an acoustics signal over the frequency range from 1 to $100 \mathrm{kHz}$.

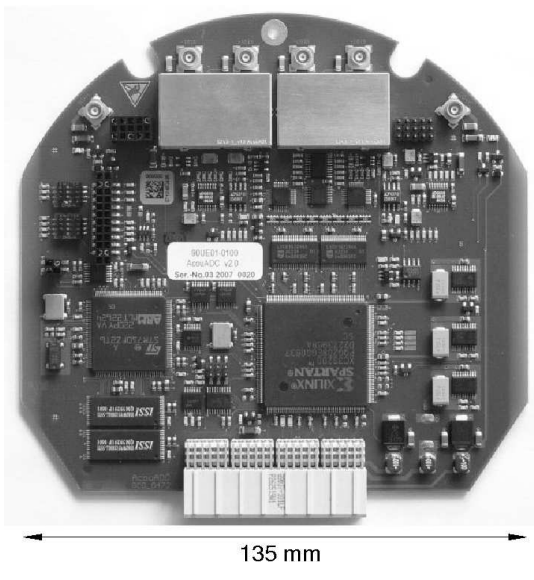

Fig. 5. An AcouADC board.

The analogue filter suppresses frequencies below $4 \mathrm{kHz}$ and above $130 \mathrm{kHz}$. The high-pass part cuts into the trailing edge of the low frequency noise of the deep-sea acoustic background [9] and thus protects the system from saturation. The low-pass part efficiently suppresses frequencies above the Nyquist frequency of $250 \mathrm{kHz}$ for the maximum sampling rate of $500 \mathrm{kSamples}$ per second (kSPS). Within the passband, the filter response is essentially flat with a linear phase response.

The digital part of the AcouADC board digitises and processes the acoustic data. It is designed to be highly flexible by employing a micro controller $(\mu \mathrm{C})$ and a field programmable gate array (FPGA) as data processor. The $\mu \mathrm{C}$ can be controlled with the onshore control software and is used to adjust settings of the analogue part and the data processing. Furthermore, the $\mu \mathrm{C}$ can be used to update the firmware of the FPGA in situ.

The digitisation is done by one 16-bit ADCs for each of the two input channels. The digitised data from the two channels is read out in parallel by the FPGA and further processed for transmission to the DAQ board. The DAQ board then handles the transmission of the data to the onshore data processing servers.

In standard mode, the digitised data is downsampled to $250 \mathrm{kSPS}$ by the FPGA, corresponding to a downsampling by a factor of 2 . Hence the frequency spectrum of interest from 1 to $100 \mathrm{kHz}$ is fully contained in the data.

Onshore a dedicated computer cluster is used to process and store the acoustic data arriving from the storeys and to control the offshore DAQ. This is discussed below.

\subsection{Onshore Data Processing}

AMADEUS follows the same "all data to shore" strategy as ANTARES; the offshore data arrives via the TCP/IP protocol at a Gigabit switch in the ANTARES control room, where the acoustic data is separated from the standard ANTARES data and routed to the acoustic computer cluster.

The cluster currently consists of four servers of which two are used for online data filtering. The filtering has the task of reducing the raw data rate of about $1.5 \mathrm{~TB} /$ day to about $15 \mathrm{~GB} /$ day for storage. The filter schemes that are currently implemented are described in [10]. Furthermore, all components are scalable which makes the system extremely flexible. Additional servers can be added or the existing ones can be replaced by the latest models if more sophisticated filter algorithms are to be implemented. In principle it is also possible to move parts of the filter into the FPGA of the AcouADC board, thereby implementing an offshore trigger which reduces the size of the data stream sent to shore.

Just like ANTARES, AMADEUS can be con- 
trolled via the Internet from essentially any place in the world and is currently operated from Erlangen. Data are centrally stored and are available remotely as well.

\section{The System Response}

Every individual component of the complete data taking chain was calibrated in the laboratory prior to deployment.

For each hydrophone and each channel of the AMs, the sensitivity was measured up to $100 \mathrm{kHz}$ as a function of the azimuthal and polar angle.

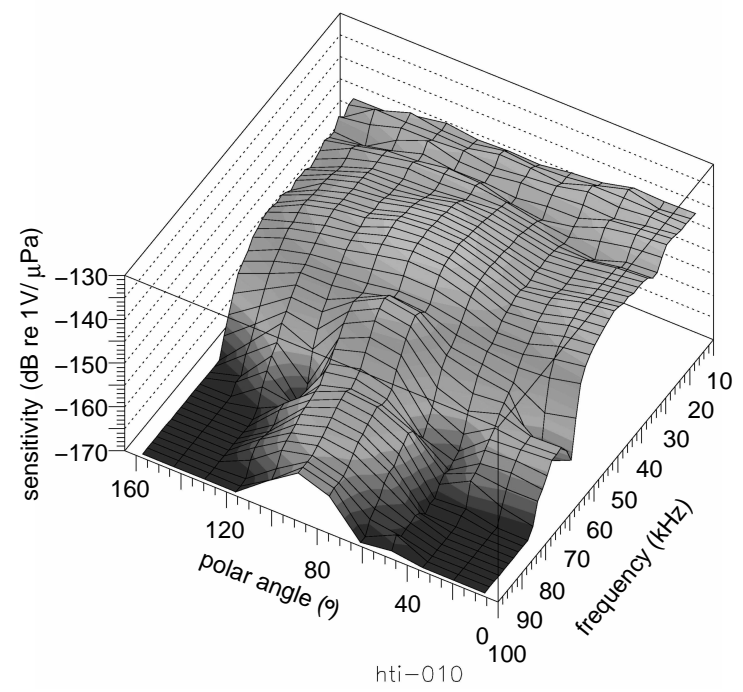

Fig. 6. Sensitivity of a typical AMADEUS hydrophone as a function of frequency and polar angle. $0^{\circ}$ corresponds to the direction opposite to the cable junction.

The sensitivity measurements were done with a calibrated sender stimulated by a Gaussian voltage pulse. The signal recorded by the acoustic sensor and the stimulating voltage pulse were transformed into the frequency domain, divided and corrected for the sender characteristics. As a result, the corrected sensitivity in $\mathrm{dB}$ re $1 \mathrm{~V} / \mu \mathrm{Pa}$ is obtained as a function of frequency for a given orientation of the sensor with respect to the sender.

For a given frequency, the sensitivity for all acoustic sensors is essentially flat as a function of the azimuthal angle on a $3 \mathrm{~dB}$ level. The sensitivity as a function of frequency and polar angle for a typical hydrophone is shown in Fig. 6 [7]. The highest sensitivity is recorded in the direction perpendicular to the longitudinal axis of the hydrophone, i.e. in the horizontal plane within an Acoustic Storey.
Furthermore, using various input stimuli at the differential analogue input connectors of the AcouADC board, the transfer function of the complete chain consisting of the electronic components forming the analogue filter and amplification stages was measured and parameterised. The digital filter that is applied in case of downsampling adds an additional, frequency-independent time delay.

In Fig. 7, a bipolar input signal is compared to the signal measured at the input of the ADC and to the result of the parameterisation of the system behaviour. The agreement is very good.

The gains of the analogue amplification were calibrated and found to agree to better than $10 \%$ with their nominal values. The system stability was checked both in the laboratory and in situ and was found to be excellent. All further relevant characteristics of the ADCs were investigated and are in full compliance with the requirements.

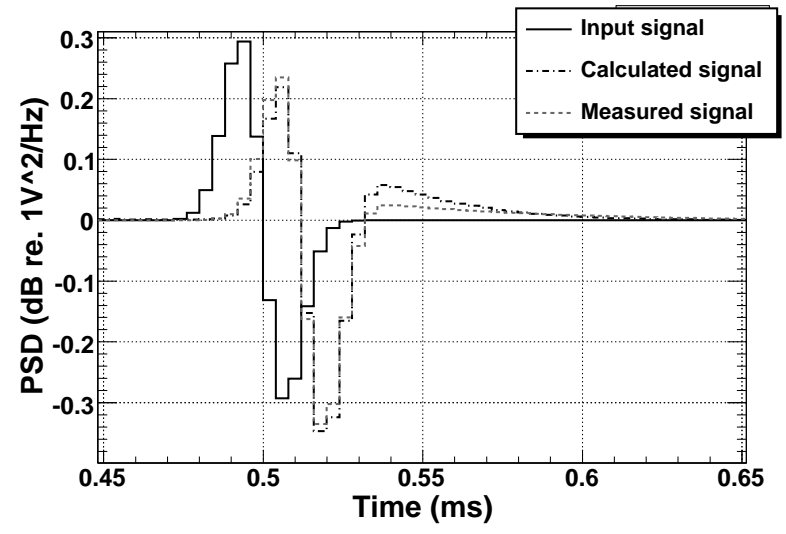

Fig. 7. A neutrino-like bipolar input signal to one of the analogue inputs of an AcouADC board, the signal recorded at the input of the ADC and the expected signal according to the parameterised transfer function. The measurement was done for a nominal gain factor of 1 .

\section{First Results}

\subsection{Ambient Noise Measurements}

The ambient noise level in the frequency range from about $200 \mathrm{~Hz}$ to $50 \mathrm{kHz}$ in the deep sea is assumed to be mainly determined by the agitation of the sea surface, i.e. by waves, spray and precipitation [11]. To verify these assumptions, the correlation between the weather and the RMS of the ambient noise recorded by AMADEUS was investigated. Weather data at the Hyères airport at the French 
coast, about $30 \mathrm{~km}$ north of the ANTARES site, is continuously logged by the AMADEUS onshore software. New data is made available every hour.

An upgrade of the study performed in [12] is shown in Fig. 8. Each point in the figure represents the RMS noise of a 10 s sample of data, calculated by integrating the power spectral density (PSD) in the frequency range from 1 to $50 \mathrm{kHz}$. The correlation between the wind speed and the hydrophone noise is found to be about $80 \%$. The results are consistent with those reported for other deep-sea sites $[13,14]$.

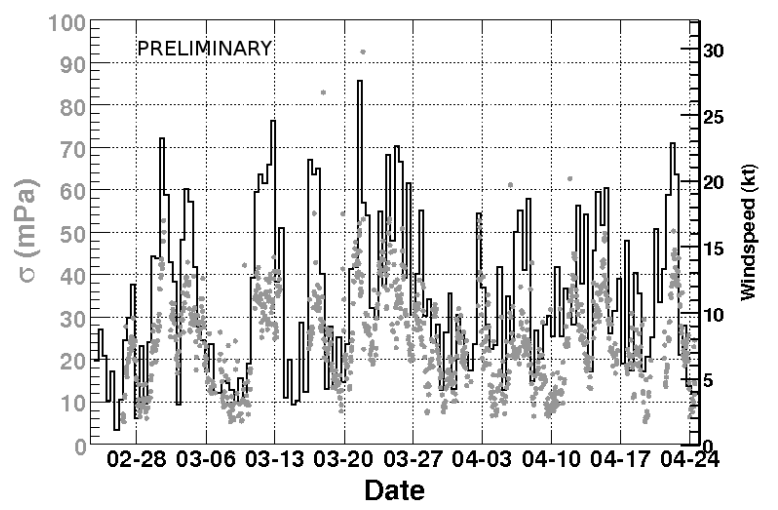

Fig. 8. RMS noise recorded by a representative hydrophone (dots) and wind speed (solid line) as a function of time, showing the correlation between the two quantities. Wind speed is given in knots.

Furthermore, the ambient noise was investigated for different sea states corresponding to different wind speeds as shown in Fig. 9. The ambient noise, characterised by the PSD, can be clearly seen to increase with the sea state. Shown is also the expected behaviour, as described by the Knudsen curves [9], which however were measured in shallow water. For the deep sea data from AMADEUS, one observes a stronger roll-off of the PSD with increasing frequency than predicted. This is qualitatively in agreement with the measurements presented in [13].

The decreasing of the PSD for frequencies smaller than about $4 \mathrm{kHz}$ cannot be unambiguously interpreted at the time of the writing of this document. It is possibly caused by effects stemming from the preliminary calibration of the hydrophone or the weighting of the directional sensitivity used to calculate the overall sensor sensitivity.

\subsection{Positioning of Acoustic Storeys}

A further important measurement with the AMADEUS system is the accurate determination

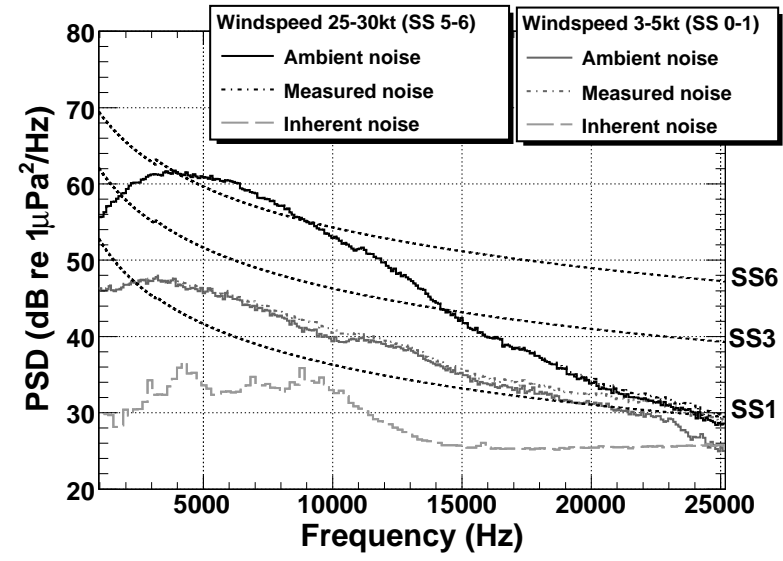

Fig. 9. Power Spectral Density (PSD) as measured for two time slices of $10 \mathrm{~s}$ length for two different conditions of the sea (sea states (SS) 0 to 1 and 5 to 6 , respectively) with a representative hydrophone. Shown are also the Knudsen curves [9] for 3 different sea states, which correspond to the expected PSDs. The ambient noise is the difference of the noise recorded in the deep sea and the inherent noise of the system, measured prior to deployment. Wind speed is given in knots.

of the relative positions of the Acoustic Storeys within the ANTARES detector (positioning). The ANTARES positioning system [15] uses transceivers (so-called pingers) at the BSS (cf. Sec. 2.2) of each line in combination with 5 acoustic receivers (positioning hydrophones) arranged along each detection line. The pingers emit tone bursts at 9 well-defined frequencies between $44522 \mathrm{~Hz}$ and $60235 \mathrm{~Hz}$ which can be used by AMADEUS to determine the positions of the Acoustic Storeys by means of triangulation.

With 6 hydrophones, a complete reconstruction (position and three angles) of each Acoustic Storey can be done using the pinger signals. This is not only an interesting task on its own, but is in fact important for an accurate reconstruction of the positions of unknown sources as will be described below. The data recorded by the Compass boards (cf. Sec. 3.2) allow for cross checks of the results.

Figure 10 shows the signal emitted by one pinger as recorded by two sensors in the AMs of the lowermost storey and by two hydrophones of the central storey of Line 12 . One can clearly observe the different arrival times of the signal (corresponding to different travel times of the sound from the pinger) between the storeys. For the two sensors of each storey shown as an example, the smaller differences in arrival times are also clearly visible.

Work on two methods for the positioning is cur- 


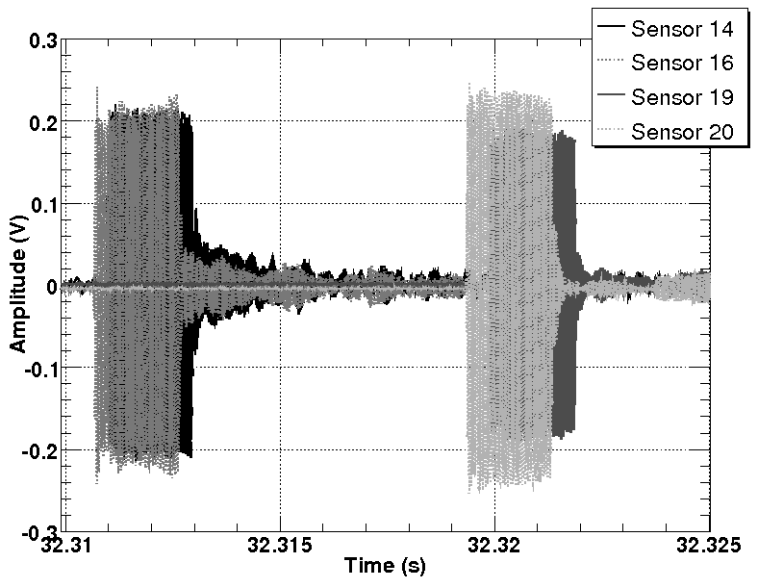

Fig. 10. Pinger signals received by four sensors in two different Acoustic Storeys on Line 12. The sensors in the lowermost Acoustic Storey (holding AMs, sensors 14 and 16) receive the signal earlier than those in the storey above (holding hydrophones, sensors 19 and 20).

rently in progress: First, the differences between the absolute time of signal emission and reception from several pingers are used to reconstruct the position of each acoustic sensor individually. Second, only the differences in arrival times of a pinger signal in the 6 acoustics sensors of a storey are used to reconstruct the direction of a pinger signal. Then the reconstructed directions are matched with the pinger pattern on the sea floor. The second method employs the same algorithms used for position reconstruction of unknown sources.

\subsection{Position Reconstruction of Sources}

Position reconstruction of acoustic point sources will be done by first reconstructing their direction from individual storeys and then combining the reconstructed directions from three or more storeys.

To find the direction of point sources, a beam forming algorithm is used. It is designed to reconstruct plane waves, which for the geometry of a storey is a reasonable assumption for sources with distances $\gtrsim 100 \mathrm{~m}$. Details can be found in [16].

\section{Conclusions and Outlook}

The AMADEUS system, which is dedicated to the investigation of acoustic neutrino detection techniques, has been successfully installed and operated within the ANTARES detector. Except for its small size, the system has all features required for an acoustic neutrino telescope and hence is excellently suited for a feasibility study of a potential future large-scale acoustic neutrino telescope.

AMADEUS can be used as a multi purpose device for studies of neutrino detection techniques, position reconstruction, and marine research.

First results were presented which demonstrate the potential of AMADEUS, covering the measurement of ambient noise, positioning of the acoustic storeys and position reconstruction of sources.

\section{Acknowledgements}

This study was supported by the German government through BMBF grant 05CN5WE1/7. The author wishes to thank the organisers of the ARENA 2008 workshop for their efforts.

\section{References}

[1] G.A. Askariyan, Sov. J. At. En. 3 (1957) 921.

[2] G.A. Askariyan, B.A. Dolgoshein et al., Nucl. Instr. Meth. 164 (1979) 267.

[3] J.G. Learned, Phys. Rev. 19 (1979) 3293.

[4] Acorne Coll., S. Bevan et al., preprint arXiv:0704.1025 [astro-ph], 2007.

[5] V. Bertin on behalf of the ANTARES Coll., these proceedings.

[6] G. Anton et al., Astropart. Phys. 26 (2006) 301.

[7] C. Naumann, Ph.D. Thesis, Univ. Erlangen-Nürnberg, FAU-PI4-DISS-07-002, 2007.

[8] ANTARES Coll., J.A. Aguilar et al., Nucl. Inst. Meth. A 570 (2007) 107.

[9] R.J. Urick, Principles of Underwater Sound, Peninsula Publishing, Los Altos, USA, 1983.

[10] M. Neff et. al., these proceedings.

[11] R.J. Urick, Ambient Noise in the Sea, Peninsula Publishing, Los Altos, USA, 1986.

[12] K. Graf, Ph.D. Thesis, Univ. Erlangen-Nürnberg, FAU-PI1-DISS-08-001, 2008

[13] N. Kurahashi and G. Gratta, Accepted by Phys. Rev. D; preprint arXiv:0712.1833 [physics.ao-ph], 2007.

[14] NEMO Coll., submitted to Deep Sea Research I; preprint arXiv:0804.2913 1 [astro-ph], 2008.

[15] M. Ardid on behalf of the ANTARES Coll., Proc. VLVnT08, to be published in Nucl. Instr. Meth. A.

[16] C. Richardt et. al., these proceedings. 